\title{
Las políticas públicas y el fortalecimiento de los emprendimientos en Jipijapa-Ecuador
}

\section{Public policies and the strengthening of enterprises in Jipijapa- Ecuador}

\author{
MSc, Zoila Mariana Delgado Gutiérrez ${ }^{1}$ \\ zoila.delgado@unesum.edu.ec \\ MSc, Gary Orlindo Vázquez Ponce ${ }^{1}$ \\ gary.vasquez@unesum.edu.ec \\ MSc, Amparo Bienvenida Baque Morán ${ }^{1}$ \\ amparo.baque@unesum.edu.ec \\ MSc, Gino Iván Ayón Ponce ${ }^{1}$ \\ ginoayon1@hotmail.com \\ MSc, Jenniffer Adriana Ponce Baque ${ }^{1}$ \\ jen_adri@hotmail.com
}

Recibido: 1/12/2017, Aceptado: 1/02/2018

\begin{abstract}
RESUMEN
Hoy en día el emprendimiento ha tomado un rol muy importante y se convierte en un tema de gran relevancia para el desarrollo de planes, programas y proyectos, cuya finalidad es crear nuevas empresas y negocios en la Zona Sur de Manabí, desde esta perspectiva se potencia la economía de un país. La metodología utilizada fue una investigación exploratoria-descriptiva, se utilizó como herramientas de trabajo la encuesta y la entrevista a expertos en el tema. Los resultados demuestran algunas debilidades en los emprendimientos desarrollados por las políticas públicas a través del Gobierno Autónomo Descentralizado del cantón Jipijapa. El emprendimiento, no solo es de ideas, es de creatividad e innovación, es como un abanico de negocio generado por las políticas públicas, para el fomento y desarrollo de las micro, pequeñas y medianas empresas, que fortalece la competitividad, asociatividad y encadenamiento productivo, en aras de contribuir al crecimiento y desarrollo económico del país.
\end{abstract}

Palabras clave: Emprendimiento, ideas de negocios, desarrollo empresarial

\footnotetext{
${ }^{1}$ Docente de la Universidad Estatal del Sur de Manabí. Ecuador
}

Revista científica Ciencia y Tecnología Vol 18 No 18 págs. 33-44 http://cienciaytecnologia.uteg.edu.ec 


\begin{abstract}
Nowadays, entrepreneurship has taken on a very important role and becomes a topic of great relevance for the development of plans, programs and projects, whose purpose is to create new businesses and businesses in the South Zone of Manabí. The economy of a country. The methodology used was an exploratory-descriptive investigation. The survey and the interview with experts on the subject were used as work tools. The results show some weaknesses in the undertakings developed by public policies through the Autonomous Decentralized Government of the Jipijapa canton. Entrepreneurship is not only about ideas, it is about creativity and innovation, it is like a range of business generated by public policies, for the promotion and development of micro, small and medium enterprises, which strengthens competitiveness, associativity and productive chain, in order to contribute to the growth and economic development of the country.
\end{abstract}

Keywords: Entrepreneurship, business ideas, business development

\title{
Introducción
}

El contenido de este artículo muestra resultados de gran valía de la investigación denominado las políticas públicas y el fortalecimiento de los emprendimientos en la Zona Sur de Manabí, a través de esto se demuestra que hay una necesidad grande de mejorar la economía de estos sectores. Por este motivo se plasma que el emprendimiento empresarial, es la generación de nuevas ideas de negocio, también considerando los conceptos más relevantes que potencian la innovación y la creatividad.

El Ecuador es reconocido a nivel latinoamericano como un país competitivo, esto debido a los esfuerzos empresariales y fiscales en el campo de la agricultura, industria, comercio y por su ubicación geográfica. Es por eso que el emprendimiento en nuestro país ha tenido participación activa en la economía; fortaleciendo el comercio en la región. Los factores que motivan el inicio del emprendimiento son: sentido de superación; gusto por el trabajo realizado; confianza en sí mismo; apoyo familiar; y, conocimiento técnico del negocio.

En el caso nacional, la Constitución del Ecuador, en su artículo 85, se refiere a las políticas públicas como instrumentos para garantizar los derechos de los ciudadanos que están reconocidos en la misma carta magna (Asamblea Nacional del Ecuador, 2008, p. 62); es decir, que las políticas ecuatorianas son necesarias siempre y cuando existan sujetos que exijan sus derechos y que, por lo tanto, demanden del Estado determinadas prestaciones que consideran vitales para su accionar.

La originalidad de este trabajo se fundamenta en estudios de gran significatividad, como es lo manifestado por (Martín \& Picazo, 2011) que realizó un estudio empírico en donde demuestran una relación positiva directa entre el emprendimiento y el crecimiento económico, pero, condicionado este emprendimiento a una coyuntura y un ambiente propicio para el desarrollo de las empresas. Acá se visualiza la importancia de formular políticas efectivas para promover los emprendimientos, que impulsen seguidamente el crecimiento económico (p. 22). 


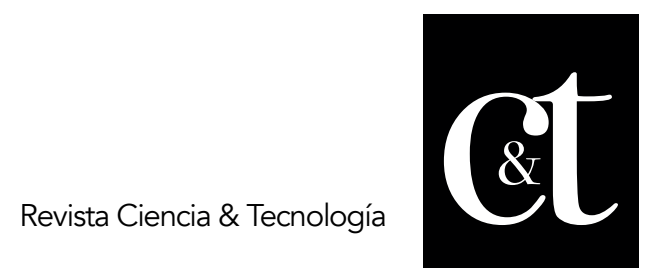

No. 18, 30 de abril de 2018

ISSN impreso: 1390 - 6321

Abondono se refiere frente a esto: "Si se espera lograr un impacto económico positivo a partir de la creación y fortalecimiento de empresas por parte de los nuevos emprendedores, se requiere una política pública que promueva y fortalezca el emprendimiento" (Abondono, 2010, p. 49).

En la misma línea de ideas, Rivas (2014), citado por la (Cámara de Comercio, Secretaria de Tic y competitividad, Universidad Autónoma de Manizales, 2015) argumenta la importancia de fomentar, a través de la política pública, el emprendimiento dinámico en las empresas, debido a la existencia de imperfecciones o fallas en el mercado. Pero para esto, es necesario en primera instancia identificar aquellas fallas, para posteriormente determinar las intervenciones de política pública más optimas:

Las implicaciones de estas fallas afectan la actividad emprendedora de diversas formas y a través de distintos canales, por lo que es conveniente identificar adecuadamente la forma en que operan, para poder diseñar intervenciones públicas que ayuden de manera efectiva a solucionar o mitigar esos problemas. Vale la pena mencionar que los niveles de actividad emprendedora no sólo se pueden ver afectados por la influencia de fallas de mercado, sino también por efecto de la propia intervención pública (regulaciones, estructura impositiva, etc.). Una forma de ordenar, en este campo, la elaboración de políticas públicas es identificar la forma en que las fallas mencionadas afectan los factores que impulsan el emprendimiento en una economía. De acuerdo a estos factores, se pueden distinguir espacios de intervención pública ya sea como parte directa de una política de fomento al emprendimiento o bien como mejoramiento de las condiciones claves para su desarrollo (p. 5).

Concluyentemente, el emprendimiento favorece el desarrollo social, económico, individual y personal, es decir no solo ayuda a solucionar la problemática macroeconómica del país, tales como el desempleo, la pobreza y la desigualdad, sino que contribuye a que los demás sectores de la economía del país crezcan.

\section{Las políticas públicas}

Las políticas públicas deben mantener una estrecha relación entre Estado -Sociedad, ya que son el componente para la solución de los problemas, su implementación permitirá a la sociedad obtener repuesta del Estado, así como su involucramiento en ámbitos gubernamentales.

Por lo tanto, las políticas públicas cumplen los objetivos que de una u otra manera la sociedad les impone por medio de sus necesidades insatisfechas, es por ello que el accionar de las instituciones que conforman el Estado deberá ser más efectivo en el momento de la toma de decisiones y por ende en la solución de problemas públicos (Roth, 2007).

Políticas públicas de apoyo al emprendimiento

Las políticas de apoyo al emprendimiento nacieron principalmente como reproducción de las acertadas medidas que tomaron los "Tigres Asiáticos" en sus modelos 


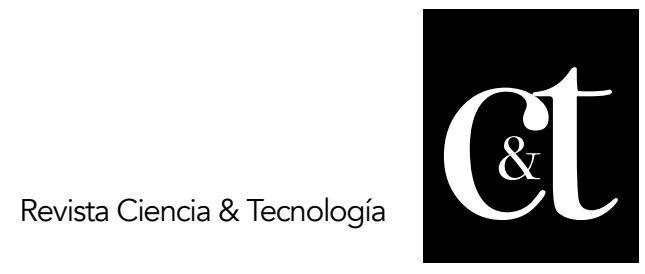

No. 18, 30 de abril de 2018

ISSN impreso: 1390 - 6321

económicos de desarrollo entre 1960 y 1990 . Dichas estrategias de desarrollo se orientaron a la sustitución de importaciones, a la promoción de la inversión privada, a una fuerte inversión y un marcado proteccionismo al sector industrial; sus resultados, permitieron apreciarla contribución de la pequeña empresa a tasas de crecimiento constantes en el PIB, exportaciones, empleo, entre otros (Held, 1995, págs. 7, 47) citado por (Beck, Demirgüç-kunt, \& Levine, 2005, págs. 20-26).

Para Hugo Kantis, el análisis de políticas de emprendimiento a nivel internacional tiene tres criterios en los que se ha justificado su creación: incrementar la base de futuros proyectos, llevar a la realidad los proyectos existentes y acelerar el crecimiento de empresas jóvenes. En este sentido, las políticas que mejores resultados han obtenido son aquellas que intervinieron en las tres dimensiones de manera paralela (Kantis \& Menéndez, 2012, p. 65).

\section{Políticas públicas sobre emprendimiento}

Una política pública se orienta a la consecución de los fines del Estado en pro del bienestar y desarrollo de una sociedad; está enfocada a lograr el equilibrio en la misma, en la promulgación de los objetivos y metas de los gobiernos. Según AndréNoél (2007), que retoma a Meny y Thoenig (1992), una política es "la acción de las autoridades públicas en el seno de la sociedad" (p. 25). Este concepto se refuerza con la opinión de Salazar, quien plantea que toda política pública se relaciona con "las sucesivas acciones del Estado frente a situaciones socialmente problemáticas" (Salazar, 1994, citado por Buitrago, 2014, p. 10).

Peters menciona que una política pública es "el conjunto de actividades de las instituciones del gobierno, actuando directamente o a través de agentes y que van dirigidas a tener una influencia determinada sobre la vida de los ciudadanos" (Peters, 1982 , p. 142). Lo más importante en una política pública es el impacto que se puede dar a partir de la implantación de planes, programas y proyectos que la soporten, por eso se espera que los efectos sean positivos y contribuyen a solucionar las necesidades de la población de manera general.

Definitivamente, el directo responsable de la implementación de políticas públicas en una sociedad es el Estado, y es el encargado de ejecutar y hacer que todas las acciones tengan un impacto, en lo posible positivo, para hacer que se le brinden a la sociedad las garantías necesarias para solucionar todas las problemáticas y todas las necesidades. De acuerdo con Olavarría, una política pública es la "intervención del Estado expresada en un conjunto de decisiones interrelacionadas, orientada a resolver, o aminorar, un problema público y que sigue un proceso formal, que lleva a dictar documentos formales, que expresan las decisiones y un proceso político que muestra la confrontación de intereses" (Olavarría, 2007, p. 44).

Políticas públicas de emprendimiento, forma de promover la cultura emprendedora De acuerdo con Ortega (2012) citado por Pérez, Zárate, Díaz, Martínez, \& Vargas (2013), "pocas acciones de política económica generan, en plena crisis, tanta unanimidad como el apoyo a los emprendedores. Pero no es algo nuevo, los gobiernos con éxitos y fracasos llevan más de cien años intentando ayudar a los emprendedores luchando contra los fallos de mercado (o de gobierno) que les impiden nacer y crecer". 


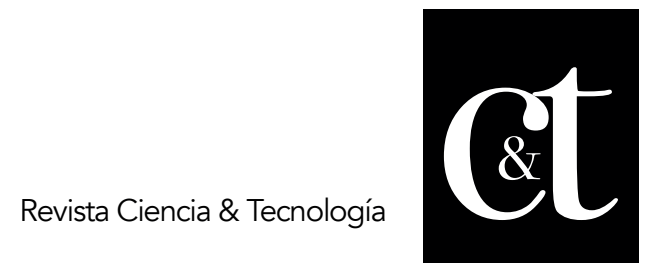

No. 18, 30 de abril de 2018

ISSN impreso: 1390 - 6321

Por años los gobiernos han intentado brindar apoyo y propiciar emprendimiento en la sociedad, pero las crisis y los problemas económicos frenan no solo las acciones gubernamentales, sino las ideas emprendedoras de los individuos.

Para poder implementar acciones a favor del emprendimiento en la sociedad, es necesario identificar por medio de estudios exhaustivos las oportunidades que ofrece el entorno, ya que las características sociales, culturales, ambientales, religiosas y económicas, juegan un papel preponderante al momento de iniciar una nueva empresa. Uno de los objetivos de las acciones del gobierno, según Pereira (2012), debe ser "identificar políticas que puedan favorecer el incremento en la calidad y en la cantidad de la actividad emprendedora".

Ospina y Rodríguez (2007) mencionan que se requiere "de política pública acorde con las necesidades de los empresarios y tendiente a equiparar los requisitos burocráticos, con los recursos de que disponen". Involucrar políticas públicas a las necesidades sociales referentes al emprendimiento, es importante ya que esto implica tener en cuenta las diferencias de los individuos y de los requerimientos que exige el entorno para poder crear ideas de negocio.

\section{Actividad emprendedora}

La actividad emprendedora tiene su importancia dentro del crecimiento económico, social, y la generación de empleo, en la implantación de nuevos mercados, pero por lo general estos mercados son informales, o terciarios. "El emprendimiento lo ejercen personas o grupos que desarrollan actividades económicas en pequeña escala con el objeto de satisfacer necesidades generando ingresos y empleo entre sus integrantes". (Asamblea Nacional del Ecuador, Ley Orgánica de la Economía Popular y Solidaria, 2011).

La actividad emprendedora de la sociedad Jipijapense se orienta principalmente a generar empleo y desarrollo a los grupos vulnerados, mejorar la calidad de los servicios, fomentar prácticas éticas en los mercados y sensibilizar a nuevos empresarios. La mayoría de emprendedores ( $86 \%)$ se dedican a su negocio propio, por el contrario, hay emprendedores más conservadores $(14 \%)$, que realizan de manera paralela otras actividades, que garanticen un flujo seguro de ingresos.

\section{Factores que inciden en la Actividad Emprendedora}

Los factores están presentes antes, durante y después del ciclo emprendedor, así como también mencionan tres factores fundamentales que se lleva a cabo en todo el proceso emprendedor: En primer lugar, el rol de las familias, dada su influencia desde las etapas tempranas es decir la estructura social, en segundo lugar, las instituciones educativas que tratan de fortalecer el desempeño de los emprendedores a través de su apoyo institucional y por último la estructura productiva, (capital social) constituye el ámbito en el que se completa el proceso de desarrollo de capacidades (Kantis H. \& Menéndez, 2012, p. 265).

En Ecuador se identifica factores internos y externos que pueden ser tanto positivos como que buscan, introducir el desarrollo de la actividad emprendedora mediante la combinación de los conocimientos personales, innovación y el aspecto 


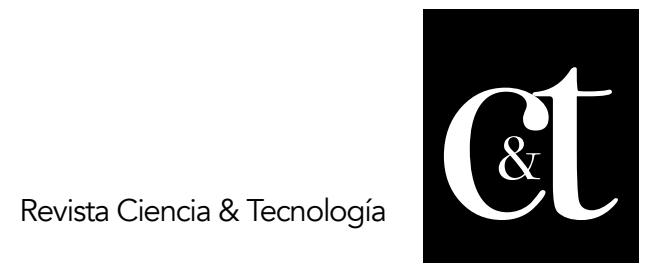

No. 18, 30 de abril de 2018

ISSN impreso: 1390 - 6321

institucional, fortaleciendo el desarrollo económico y la generación de empleo. Para ello se pone en manifiesto que depende exclusivamente de la percepción de los emprendedores, identificar si dichos factores internos y externos inciden positiva 0 negativamente al desarrollo de la actividad emprendedora, porque estos pueden variar significativamente de acuerdo al criterio de cada persona.

En lo que respecta, los factores internos positivos y negativos que rodean a los emprendedores pueden presentarse como ventajas y/o debilidades, que se encuentran dentro de la estructura social de cada individuo y "están relacionados principalmente a características actitudinales de las personas, aspecto familiar, conocimientos etc." (Araque, 2009).

\section{El emprendedor ecuatoriano}

Caracterizar al emprendedor ecuatoriano es importante por ser el eje al cual se dirigen todos los esfuerzos de la política pública. Cabe mencionar que no existe una literatura extensa sobre emprendimiento de carácter dinámico en el país, por lo tanto, los resultados abarcan también negocios que pueden ser considerados tradicionales. Es importante iniciar esta caracterización analizando las cualidades individuales del emprendedor ecuatoriano promedio, en las que se lo identifica como una persona confiada, organizada, informal, competitiva, adversa al riesgo, que busca estabilidad, que tiene experiencia en la actividad y está motivada por la autorrealización, la contribución a su sociedad y la aplicación de sus conocimientos (Villaroel y otros, 2003, citado por Escuela Politécnica del Litoral, 2013, pp. 22-35).

Wilson Araque, en su investigación que determina el perfil del emprendedor ecuatoriano complementa lo mencionado, lo caracteriza como una persona con estudios formales, que prefiere adquirir experiencia laboral con los años, que no se desarrolló empresarialmente a partir de un plan de negocios y mantiene empresas jóvenes, generalmente creadas por necesidad y deseos de independencia (Araque, 2009, pgs. 43-70).

La tipología de empresa promedio se muestra como un negocio familiar, generado en su mayoría por necesidad y con dificultades en el proceso de instauración. Tiene un promedio de 7 años de funcionamiento, está enfocado al mercado local y con mayor direccionamiento hacia áreas de comercio y servicios; su fuente de financiamiento prioritaria son los recursos propios (Ordeñana \& Arteaga, 2012, pp. 4-8).

Sus problemas principales son la falta de recursos, la percepción de falta de apoyo, problemas administrativos empresariales internos, legislación inadecuada, y la escasa investigación científica y tecnológica que realiza al interior de su negocio (Flor, 2011, p. 263).

Otro factor de discreción es el nivel socio económico del emprendedor. Según Xavier Ordeñana y María Elizabeth Arteaga, las personas provenientes de hogares de medianos o altos ingresos económicos tienen mayores probabilidades de desarrollar un emprendimiento dinámico. Un porcentaje minoritario, proviene de un hogar de escasos recursos económicos (Ordeñana \& Arteaga, 2012, pp. 13-23). 


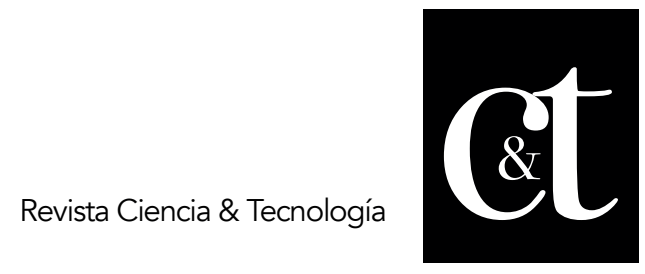

No. 18, 30 de abril de 2018

ISSN impreso: 1390 - 6321

En cuanto a emprendimiento dinámico, María Elizabeth Arteaga y María Virginia Lasio, investigadoras de la ESPAE, realizaron una investigación donde determinaron un indicador de discreción en este concepto, del total de empresas ecuatorianas encuestadas y analizadas en su estudio, aproximadamente el $17 \%$ se consideraron dinámicas, entendiendo este término como aquellas empresas con un incremento mínimo de ventas del $10 \%$ en los primeros 5 años, y un crecimiento de empleados del $20 \%$ después del tercer año (Arteaga \& Lasio, 2009, pp. 1-17).

El emprendedor promedio a nivel latinoamericano se asemeja en gran medida al caso ecuatoriano, en especial en su diferenciación por ingresos económicos y en sus cualidades individuales; sin embargo, hay criterios importantes de disparidad como el caso de los salarios más representativos, la mejor explotación de negocios con componente innovador, el enfrentamiento a menores problemas de gestión como el acceso a clientes, proveedores, financistas, a fuerza laboral calificada (Gluzmann, Jaume, \& Gasparini, 2012 pp. 101-104), en breves rasgos tienen mayor desarrollo que sus pares ecuatorianos.

\section{Metodología}

Para mayor objetividad del proceso investigativo se realizó un estudio cualitativo en el cual se implementaron entrevistas, se analizaron informes e información relevante de diversas fuentes, que permitieron plasmar el bosquejo de las políticas públicas de emprendimiento en el cantón Jipijapa. En base a esto se generó un acercamiento y aproximación a la realidad de los emprendimientos. De esta manera, el análisis de los datos dio la posibilidad de tener los diferentes puntos de conexión que se tienen en cuenta en cualquier contexto.

Una demostración tan palpable, fue la obtenida en la recopilación de datos a través de las diferentes fuentes, para poder establecer el impacto de las políticas públicas en Jipijapa, así como el nivel de emprendimiento, el porcentaje de empresas creadas en los últimos años y los planes, programas y proyectos implementados por Gobierno Autónomo Descentralizado de la Municipalidad de Jipijapa, con la finalidad de incentivar y promover el emprendimiento y la cultura emprendedora. Además, se determinó que aspectos jurídicos y políticos han influido en el desarrollo del emprendimiento en el cantón.

Se analizó el nivel de impacto de las políticas públicas que potenciaron el emprendimiento en la sociedad, considerando a los sectores que generan más confianza en la población y cuáles son lo que menos incentivan la creación de empresa o negocios. Todo esto estuvo relacionado con la intervención y las políticas implementadas por parte del Gobierno Autónomo Descentralizado de la Municipalidad de Jipijapa y cuáles son las posibilidades de fomentar e incentivar el emprendimiento en la creación de empresa o negocios.

Otra herramienta utilizada fue la entrevista, donde se observó la confianza de las acciones gubernamentales y de las entidades responsables de promover el emprendimiento en el cantón Jipijapa, analizando de qué manera el GAD fortalece la cultura empresarial y como desarrolla los planes, programas y proyectos planteados para motivar a las personas a crear empresa, porque el dinamismo empresarial y comercial es el motor de la actividad económica de toda sociedad. 


\section{Resultados}

El trabajo de investigación sustenta este artículo en base a las normas jurídicas, que fundamentan las políticas públicas.

Para fundamentar los resultados del proceso investigativo con mayor exactitud, se consideró datos del INEC. Desde esta perspectiva es imprescindible destacar que la actividad comercial y la provisión de servicios se concentran en la cabecera cantonal Jipijapa y en menor proporción en las cabeceras parroquiales, así lo ratifican los datos estadísticos del INEC que muestran que el $42,70 \%$ de la población ocupada se vincula al sector comercial y de servicios en general. El siguiente cuadro muestra que el cantón Jipijapa tiene un total de 1.651 establecimientos comerciales que satisfacen las necesidades de las áreas vinculadas a: manufacturas, comercio, servicios, agricultura, etc.;

Empresas por ramas de Actividades comerciales por sectores productivos - cantón Jipijapa.

En la investigación de campo se consultó a un total de 55 empresarios de diversas ramas de actividad económica en la ciudad de Jipijapa. El 56\% de las empresas consultadas pertenece al sector comercio al por mayor y al por menor, el $33 \%$ corresponde al sector servicios, el $9 \%$ al sector manufacturero y el $2 \%$ a otras actividades de agricultura, organizaciones y órganos extraterritoriales. La diversidad de los sectores económicos en la muestra permite dar un panorama ampliado y heterogéneo de percepciones en los empresarios.

Como parte de la dinámica local se puede destacar que aunque en la cabecera cantonal se encuentran los principales establecimientos comerciales y servicios la realidad nos demuestra que estas actividades no podrían conservarse activas (rentables) si no existiera la actividad agrícola dinamizada por los pequeños y medianos agricultores; ellos en los días de feria (fin de semana) visitan los mercados donde venden sus productos y se abastecen de lo que necesitan (artículos de primera necesidad, herramientas, insumos agrícolas, ropa, electrodomésticos, entre otros.), en este caso el crecimiento de los núcleos poblacionales como la cabecera cantonal depende en gran medida del éxito o fracaso de la actividad agropecuaria.

1. Cree que los emprendimientos del cantón Jipijapa son favorecidos y promovidos por el gobierno central.

En respuesta a lo mencionado en la gráfica anterior, en la actualidad un $72 \%$ de los encuestados afirma que la dinámica emprendedora en Jipijapa es favorecida y promovida por el gobierno central, en contraste anteriores que estaba en un $19 \%$ en un estudio que Catapulta realizó en 2007 y en 2011 subió a un 69\%. Esto quiere decir que se han incrementado los emprendimientos, el nivel de esfuerzos se está solidificando, y la ciudadanía reconoce los avances a favor de una cultura emprendedora.

2. Las herramientas de apoyo al emprendimiento son visibles desde hace:

Se puo verificar que, si bien los primeros pasos se dieron en el 2011, apenas hace tres años resultan visibles las herramientas de apoyo al emprendimiento en el cantón 
Jipijapa, como lo afirma el $65 \%$ de los encuestados.

3. ¿Qué le motivo a los emprendedores a crear una empresa?

Los resultados obtenidos son algo espontáneo de lo que sucede en América Latina, al igual que en el resto del mundo, más del 38\% de los encuestados del cantón Jipijapa afirman que los motivos más influyentes a crear empresas es la Autorrealización y Mejorar sus ingresos. El 18\% relativo a Mejorar sus Ingresos está relacionado con el alto nivel de emprendimiento por necesidad del cantón Jipijapa.

4. ¿Cree usted que utilizando medidas más flexibles afectarían el crecimiento de su empresa?

En cuanto a la pregunta de cómo afectaría al crecimiento empresarial si se utilizaran medidas que flexibilizaran las dificultades que se presentan, el $75 \%$ de los empresarios afirmó que estas mediadas impactarían sustancialmente el crecimiento de sus empresas.

5. ¿Ha recibido usted el apoyo de parte del GAD Jipijapa para conocimientos de programas de emprendimiento en la generación de nuevas empresas?

Esta relación puede evidenciarse en el conocimiento, por parte de emprendedores y el GAD de Jipijapa de apoyo al emprendimiento, de programas o iniciativas que apoyan la generación de nuevas empresas. El $72 \%$ de las Unidades de Emprendimiento entrevistadas manifestaron conocer dichos programas (gráfico 1).

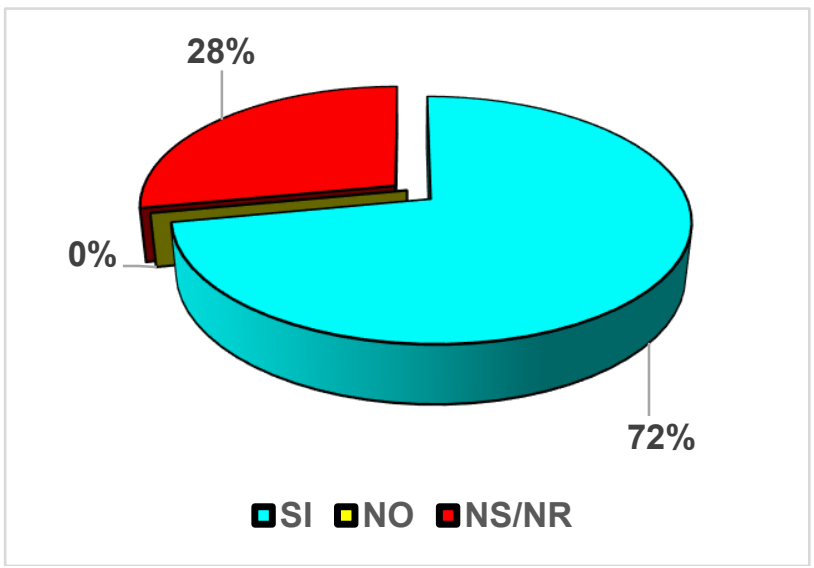

\section{Gráfico 1. Conocimiento de programas de emprendimiento} Elaborado por: Los Autores

Por último y no menos importante, en la investigación realizada se identifica la necesidad de fortalecer al interior del GAD Jipijapa que trabajen por el apoyo al emprendimiento y la creación empresarial, la cultura del emprendimiento y la innovación, en formación, integración interna y gestión de las dependencias que permita articular también a la universidad en la docencia, la investigación y la extensión en pro de generar una oferta más diversificada, innovadora y ajustada a 


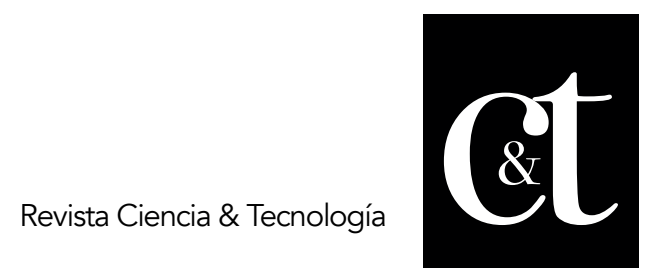

No. 18, 30 de abril de 2018

ISSN impreso: 1390 - 6321

las necesidades de los diferentes tipos de emprendimiento y emprendedores de la ciudad de Jipijapa.

\section{Conclusiones}

Haciendo énfasis en los últimos años, los avances en políticas de emprendimiento, así como el fortalecimiento de programas y proyectos para promover e incentivar las ideas de negocios y la creación de empresa. A partir de esto se ha evidenciado que se pude invertir y acceder al apoyo estatal en lo relacionado a manufactura, comercio y servicios, es decir las entidades gubernamentales están centrando sus esfuerzos en fortalecerlos. De acuerdo con esto, se puede definir que la implementación de las políticas públicas es pertinente y acorde a las necesidades de la población, aunque necesitan de estrategias de comunicación más efectivas.

Las redes de emprendimiento en otras regiones del país se han convertido en un mecanismo muy importante para trabajar con las entidades territoriales por sectores, en el enfoque de la creatividad con ideas de negocios y la creación de empresas.

Desde este enfoque se hace alusión a lo más relevante, las ideas de negocio relacionadas con el comercio que son las que mayor impacto y acogida tienen por los emprendedores en la ciudad de Jipijapa. Pero en los últimos años sectores como tecnología, turismo, hotelería, restaurantes, manufactureras y de servicios, han crecido potencialmente, generando gran confianza en la población. Algunas presentan debilidades como el limitado dinero, la falta de orientación y asesoría y el miedo a fracasar en su iniciativa de la idea de negocio.

Las políticas públicas de emprendimiento han tenido muy buen impacto en el país, y sobre todo en algunos sectores de la ciudad de Jipijapa, pero los resultados podrían ser mejores si se establecen criterios de acceso y de divulgación para la mayor parte de la población. Es evidente que los esfuerzos de las últimas décadas han sido fructíferos, pero no con los resultados que se requieren para generar mayor desarrollo en el cantón y en el país.

Concluyentemente la comunidad no tiene confianza en el Gobierno Autónomo Descentralizado de la Municipalidad del cantón Jipijapa, y de otras instituciones gubernamentales, porque la población genera, de algún modo, inconvenientes en la efectividad de los planes y programas, sus habitantes no están de acuerdo con los trámites burocráticos que tiene que realizar para crear su negocio o emprendimiento.

\section{Referencias bibliográficas}

Abondono, J. M. (2010). Sobre la política nacional de emprendimiento: un enfoque desde la competitividad. Perspectiva. Obtenido de http://www.revistaperspectiva.com/archivos/revista/No\%2023/04749\%20PERS\%200K.pdf

André-Noél, R. (2007). Políticas públicas: Formulación, implementación y evaluación. Bogotá: Ediciones Aura.

Araque, W. (2009). Perfil del emprendedor ecuatoriano. Universidad Andina Simón Bolívar. Quito: Corporación Editora Nacional. 


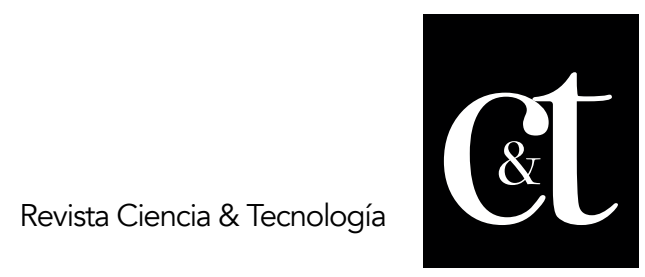

No. 18, 30 de abril de 2018

ISSN impreso: 1390 - 6321

Arteaga, M. E., \& Lasio, V. (2009). Empresas dinámicas en Ecuador: factores de éxito y competencia de sus fundadores.». Academia, Revista Latinoamericana de Administración (CLADEA), 42.

Asamblea Nacional del Ecuador (2008). Constitución de la República del Ecuador, Montecristi: Registro Oficial, Ecuador.

Asamblea Nacional del Ecuador (2011). Ley Orgánica de la Economía Popular y Solidaria.

Beck, T., Demirgüç-kunt, A., \& Levine, R. (2005). SMES Growth, and Poverty. United States: NBER Working Paper No. 11224.

Buitrago, N. J. (2014). Emprendimiento en Colombia. Administración \& Desarrollo, ISSN-e 0120-3754, Vol. 43, No. 59, 2014, págs. 7-21. Obtenido de file://C:/Users/ambam/Downloads/101-1585-1-PB\%20(2).pdf

Cámara de Comercio, Secretaria de Tic y competitividad, Universidad Autónoma de Manizales (2015). Estudio de política pública para el emprendimiento y el crecimiento empresarial en Manizales.

Escuela Politécnica del Litoral (2013). Informe GEM General Entrepreneurship Monitor Ecuador 2012. Guayaquil Ecuador.

Flor, G. (2011). Guía para crear y desarrollar su propia empresa. Quito - Pichincha: Cámara Ecuatoriana del Libro.

Gluzmann, P., Jaume, D., \& Gasparini, L. (2012). Decisiones laborales en América Latina: El caso de los emprendedores. CAF Working Papers (CAF), n 2012/06.

Held, G. (1995). Políticas de financiamiento de las empresas de menor tamaño: Experiencias recientes en América Latina. serie financiamiento para el desarrollo 34. Santiago de Chile: Comisión Económica para América Latina y El Caribe.

Kantis, J. F. \& Menéndez, C. (2012). Políticas de fomento al emprendimiento dinámico en América Latina: Tendencias y desafíos. Argentina: CAF Working Papers.

Martín, M. A., \& Picazo, M. T. (2011). La actividad emprendedora y competitividad: Factores que inciden en los emprendedores. Papeles de Europa. Europa.

Meny, I. y Thoenig, J.C. (1992). Las políticas públicas. Barcelona: Editorial Ariel.

Olavarría, M. (2007). Conceptos Básicos en el Análisis de Políticas Públicas. Documentos de trabajo. Obtenido de file://C:/Users/ambam/Downloads/1011585-1- PB\%20(2).pdf

Ordeñana, X. \& Arteaga, E. (2012). Middle-class entrepreneurship and the effect of social capital. Guayaquil: Inter-American Development Bank Working Paper No.IDB-WP-318. Guayaquil.

Ospina, D. y Rodríguez, D. (2007). Políticas públicas de micro, pequeñas y medianas empresas en el Departamento de Caldas. jurid. Manizales (Colombia), 4(2): 31 - 


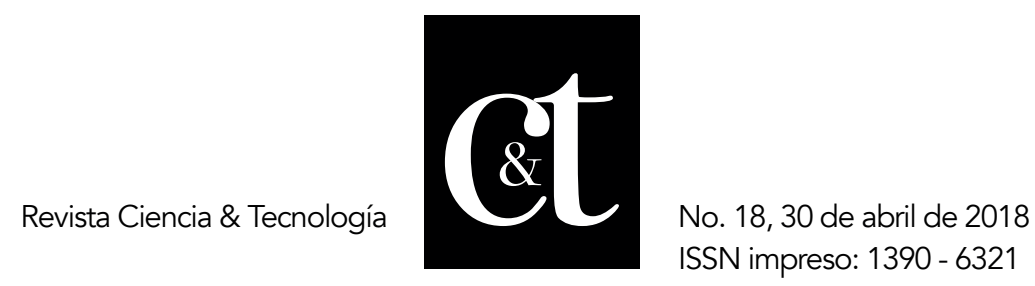

45, Julio-Diciembre 2007.

Pereira, F. (2012). Colombia Global Entrepreneurship Monitor 2011 - 2012. Ediciones Sello Javeriano, Colombia.

Pérez, U. R., Zárate, T. R., Díaz, C. N., Martínez, S. J., \& Vargas, L. J. (2013). Emprendimiento sostenible. Colombia: Dirección Gestión del Conocimiento.

Peters, B. (1982). American Public Policy. New York: FWP.

Rivas, G. (2014). El fomento al emprendimiento dinámico en países del Cono Sur de América Latina. Análisis de experiencias y lecciones de política. Banco Interamericano de Desarrollo. Instituciones para el Desarrollo. División de Competitividad e Innovación.

Roth, A. N. (2007). Política Pública. Bogotá: Aurora. 